\title{
Navier-Stokes Computations and Experimental Comparisons for Multielement Airfoil Configurations
}

\author{
W. Kyle Anderson*,Daryl L. Bonhaus*,Robert McGhee*, and Betty Walker* \\ NASA Langley Research Center \\ Hampton, VA 23681
}

\begin{abstract}
A two-dimensional unstructured Navier-Stokes code is utilized for computing the flow around multielement airfoil configurations. Comparisons are shown for a landing configuration with an advanced-technology flap. Grid convergence studies are conducted to assess inaccuracies caused by inadequate grid resolution. Although adequate resolution is obtained for determining the pressure distributions, further refinement is needed to sufficiently resolve the velocity profiles at high angles of attack.

For the advanced flap configuration, comparisons of pressure distributions and lift are made with experimental data. Here, two flap riggings and two Reynolds numbers are considered. In general, the trends caused by variations in these quantities are well predicted by the computations, although the angle of attack for maximum lift is overpredicted.
\end{abstract}

\section{Introduction}

The goal of a high-lift system is to generate as much lift as possible without separating the flow [1]. Without external devices such as wall suction, the most effective way to achieve this goal is through the use of multiple elements to manipulate the inviscid pressure distribution to reduce the pressure rise over each element [1], [2]. However, the presence of multiple elements seriously complicates analysis procedures because of important and often complex interactions between the individual elements. While inviscid analysis can be accomplished in minutes with panel methods or unstructured-grid Euler solvers, it is necessary to use viscous techniques to accurately predict the flows about these configurations. The reason for this is that although the tailoring of the flow field to prevent separation is largely achieved through circulation interactions between the elements, many viscous effects can have large influences on the pressure distributions. While these include obvious effects such as displacement thickness and separation on the surfaces, wake interactions between forward and aft elements as well as flow reversal off the surface can contribute significantly in determining the overall performance of the highlift system [3], [4], [5].

For computations on multielement airfoils, unstructured grid methods may offer a good alternative to more traditional methods of analysis. This is due in part to the decreased time required to generate grids over complicated geometries. Also, unstructured grids offer the potential to adapt the grid to improve the accuracy of the computation without incurring the penalties associated with global refinement. However, despite the advantages of unstructured grids, they are typically much slower than structured grid solvers. Also, the ability to obtain solutions through local adaptation that are comparable to those obtained through global refinement remains an area where further work is required [6]. Although work remains to fully realize their potential, much progress has been reported in computing viscous flows on unstructured grids (See for example [7], [8], [9], [10]).

The purpose of this study is to present computational results obtained with a particular unstructured grid method [11], [12]that has been applied to several flows over multielement airfoils. Comparisons between computational results and experimental data are

\footnotetext{
* Fluid Mechanics Division, member AIAA

Presented as paper 93-0645 at the Aerospace Sciences Meeting, Reno Nevada, 1993. Copyright (C) 1994 by the American Institute of Aeronautics and Astronautics, Inc. No copyright is asserted in the United States under Title 17, U.S. Code. The U.S. Government has a royalty-free license to exercise all rights under the copyright claimed herein for Governmental purposes. All other rights are reserved by the copyright owner.
}

made to assess the effectiveness of the present code, to aid in determining future directions, and to provide useful comparisons for other researchers working in this field.

\section{Symbols}

\begin{tabular}{|c|c|}
\hline$\alpha$ & angle of attack \\
\hline$b$ & span of wind tunnel model \\
\hline$c$ & $\begin{array}{l}\text { reference chord taken to be the chord of the } \\
\text { undeflected airfoil }\end{array}$ \\
\hline$C_{l}$ & lift coefficient \\
\hline$C_{p}$ & pressure coefficient \\
\hline CFL & Courant-Friedichs-Lewy number \\
\hline$M_{\infty}$ & free stream Mach number \\
\hline$q$ & magnitude of velocity \\
\hline$q_{\infty}$ & magnitude of velocity in freestream \\
\hline$R e$ & Reynolds number \\
\hline$u$ & $\begin{array}{l}\text { velocity component in direction of } \\
\text { surface-tangent vector }\end{array}$ \\
\hline$x, y, z$ & Cartesian coordinates \\
\hline$y^{+}$ & turbulent boundary-layer parameter \\
\hline$\alpha$ & angle of attack \\
\hline$\eta$ & $\begin{array}{l}\text { chordwise location on airfoil (referenced to } \\
\text { undeflected position) }\end{array}$ \\
\hline
\end{tabular}

\section{Computational Method}

The computational method used in this study is a node-based, implicit, unstructured, upwind flow solver described in reference [11]. In this code, the discretization of the convective and viscous terms is handled similarly to the method of reference [8]. The inviscid fluxes are obtained using Roe's approximate Riemann solver [13]; the viscous terms are evaluated with a Galerkin-type approximation that results in a central-difference formulation for these terms. Two different turbulence models are presently utilized in the code. These include both the Baldwin-Barth [14] model and the Spalart-Allmaras [15] model. At each time step, the equation 
for the turbulent viscosity is solved separately from the flow equations, which results in a loosely coupled solution process that allows for a relatively easy interchange of other turbulence models. Although both turbulence models have been used extensively with good success, the present study reports only results obtained with the Spalart-Allmaras model.

\section{Experimental Data}

All experimental data used in the present work have been obtained in the Low Turbulence Pressure Tunnel (LTPT) located at the NASA Langley Research Center [16]. The tunnel is a single return, closed-throat wind tunnel that obtains high Reynolds numbers by operating at pressures up to $10 \mathrm{~atm}$. The test section is $3 \mathrm{ft}$ wide by $7.5 \mathrm{ft}$ high by $7.5 \mathrm{ft}$ long. Side wall boundary suction is applied to promote two-dimensional flow [17].

Lift and moment measurements are obtained by using both a force balance and an integration of surface pressures; drag is obtained from a wake survey with a five-hole probe. The accuracy of the lift force is approximately \pm 0.5 percent when obtained from the balance [16], and the lift coefficient is estimated to be within \pm 0.03 when obtained from pressure integration. The drag coefficient is estimated to be accurate to within \pm 0.001 [18] for attached flows. Pressure coefficient distributions are obtained from pressure orifices located along the model and are accurate to within about \pm 0.030 .

For the calculations that follow, comparisons will be made with experiments obtained from two different data sets and the presentation of results is organized accordingly. A brief description is given below for each data set.

The test data is the result of a cooperative experimental program between the Douglas Aircraft Company and the NASA Langley Research Center and is reported in references [19] and [20]. Test Reynolds numbers varied between 5, 9, and 16 million. The angle of attack included a range of approximately $-4^{\circ}$ through $23^{\circ}$. The tests have been conducted without forced boundarylayer transition. The overall geometry, which is shown in figure 1 , is a three-element configuration based on an 11.55 percent thick supercritical airfoil. The slat and flap chord ratios are 14.48 percent and 30 percent, respectively, based on the airfoil chord for the undeflected position.

For the current study, the deflections of both the slat and the flap are set at $30^{\circ}$, and two different flap riggings are considered. A "rigging" refers to a combination of gap and overhang settings as defined in figure 2, and a specific rigging is assigned a letter designation. For the first configuration, denoted as 30P-30N (slat deflection $30^{\circ}$, slat rigging $\mathrm{P}$, flap deflection $30^{\circ}$, flap rigging $\mathrm{N}$ ), the flap overhang is 0.25 percent of the undeflected airfoil and the flap gap is 1.7 percent. For the second configuration, which is denoted as 30P-30AG, both the gap and overhang are 1 percent. In figure 3, a more detailed view of the flap riggings for the two configurations is shown.

The data for these configurations have been obtained from two separate tunnel entries. Because the first test considered both three- and four-element airfoils, the flap was constructed in two pieces, which were then assembled on site. During these tests, force and moment data were obtained for many flap riggings, including those discussed here. From these experiments, it was found that the $30 \mathrm{P}-30 \mathrm{~N}$ configuration exhibited a slightly higher $C_{l_{\max }}$ than did the 30P-30AG. A single-segment flap was then constructed and the $30 \mathrm{P}-30 \mathrm{~N}$ geometry was studied in more detail in a subsequent test. During this test, more detailed data, such as velocity profiles, were obtained and are presented in reference [20].

In order to make meaningful interpretations between computational and experiment results, an indication of the two dimensionality of the flowfield is necessary. Figure 4 shows experimental pressure distributions at several angles of attack obtained at two different spanwise locations on the model. The $z / b=0.5$ location corresponds to the centerline of the tunnel which has 146 pressure taps. In order to better assess the two dimensionality of the flow, several pressure taps are also present at $z / b=0.77$, which is approximately midway between the centerline of the model and the wind tunnel wall. As seen in the figure, excellent two-dimensional flow is maintained at an angle of attack of $16.2^{\circ}$ (uncorrected). Slight three-dimensional effects are present at $21.31^{\circ}$ and three dimensionality is clearly indicated at $22.25^{\circ}$.

\section{Results}

The results of the comparisons are presented below. For the computations, the CFL number has been ramped linearly from 20 to 100 over 100 iterations. The lift coefficient is constant to within about 0.1 percent of the total lift (fourth significant digit) and the pressure distributions and velocity profiles show no differences over 500 iterations when plotted.

All grids used in the present study have been generated using the grid generation procedure described in reference [21]; a sample is shown in figure 5. In this procedure, structured grids are first generated around individual components. These grids are then used to define a cloud of points that is triangulated with a stretched-Delaunay triangulation procedure to establish the connectivity relationships. Although the aspect ratios of the triangles near the surface are generally very large because of the extremely small spacing required at the wall, grids generated in this manner tend to have relatively few cells with large angles that can negatively compromise accuracy. For the grids used here, fewer than 2 percent of the angles are greater than $120^{\circ}$.

Comparisons between computed results and experimental data are presented below for the three-element advanced flap configurations (30P-30N and 30P-30AG) discussed above. The first set of results is for the $30 \mathrm{P}-30 \mathrm{~N}$ configuration at several angles of attack with a Mach number of 0.2 and a Reynolds number of 9 million. Simultaneously presented are the results of varying grid densities on computed pressure distributions and velocity profiles, which are used to ascertain the level of numerical errors in the computations.

For these studies, three grids have been utilized. The first grid, which will be referred to as the coarse grid, consists of 22,491 nodes, 524 of which lie on the surfaces of the elements. The spacing at the wall for this mesh is $4 \times 10^{-6}$ normalized to the chord length of the airfoil in the undeflected position. This spacing yields a $y^{+}$of less than 2 for the point next to the wall at the rear of a unit-length flat plate. Finer grids are obtained by simultaneously increasing the number of points in each direction to obtain as close to a uniform refinement as possible. In the current study, the number of points in each direction is increased with each refinement by a factor of roughly $\sqrt{2}$ so that the total number of nodes with each refinement is approximately doubled over the previous mesh.

With this procedure, the second mesh in this family of grids contains 49,596 nodes with 806 points on the surfaces. This mesh will be referred to in future discussions as the baseline mesh. For this mesh, the spacing at the wall is about $2 \times 10^{-6}$ and results in a $y^{+}$of less than 1 , according to flat plate estimates. In a similar 
fashion, another finer grid is generated that has 87,783 nodes with 1036 points on the solid surfaces; it is referred to here as the fine grid.

Pressure distributions on all grids are shown in figures 6 through 9 for $\alpha=-0.03^{\circ}, \alpha=8.23^{\circ}, \alpha=16.30^{\circ}$, and $\alpha=22.36^{\circ}$, respectively. As seen, the variation in the pressure distribution between the coarse grid and the other two grids is relatively small up to $\alpha=22.36^{\circ}$. However, at this angle of attack, the loading on the flap is noticeably less for the coarse grid than for the baseline and fine grids, which continue to yield very similar results. Also, an obvious discrepancy exists in the pressure distribution on the slat for an angle of attack of $8.23^{\circ}$. The cause of this is unknown, but could be attributable to a number of sources including wind tunnel wall corrections or an inaccurate representation of the separated flow under the cove which leads to higher circulation around this element. Although not shown, it is interesting to note that excellent agreement is obtained on the slat, as well as the other elements, when the point vortex correction in the far field for the computations is removed. However, numerical studies for the Euler equations with this same three-element airfoil indicate that the presence of the point vortex significantly decreases the dependence of the solution on the placement of the far-field boundary as expected. Therefore, the improved agreement obtained without the vortex is evidently fortuitous. Note also that the pressure distributions on both the main element and the flap indicate slightly higher lift than the experiment, which could also account for a higher circulation on the slat.

Computed velocity profiles at several locations along the main element and the flap are shown in figure 10 for an angle of attack of $22.36^{\circ}$. Also shown is an illustration that indicates the locations on each element where the data are obtained. Note that these locations are referenced to the airfoil coordinates in the undeflected position. Results are shown for this angle of attack because they indicate the most variation between the coarse, baseline, and fine grids. As seen, the agreement between the baseline grid and the fine grid is reasonably good on the main element but the coarse grid is clearly inaccurate. Furthermore, the trend with grid refinement is to decrease the boundary-layer thickness on the main element. The increased thickness of the boundary layer with the coarse grid is likely to be responsible for the decreased flap loading obtained on this grid and seen in figure 9 .

On the flap, the major difference between the baseline grid results and the fine grid results is in the enhanced resolution of the slat wake on the finer grid. This wake is very apparent at the station immediately downstream of the main element $(\eta=0.72)$ but quickly dissipates so that its presence is barely detectable at the location towards the back of the flap $(\eta=0.92)$. The difference in profiles between the baseline and fine grids indicates that further refinement is necessary to accurately resolve these details. Although not shown, as the angle of attack is reduced, the difference in the profiles decreases so that the baseline grid and fine grid give essentially identical results at an angle of attack of $-0.03^{\circ}$.

An additional study of grid effects has been conducted and is presented in figure 11. For this study, a grid very similar to the baseline grid has been generated with the spacing of the grid points next to the wall based on obtaining a $y^{+} \approx 10$ instead of $y^{+} \approx 1$. Note that the spacing is determined based on estimates from a flat plate at the Reynolds number in question (Re $\left.=9 \times 10^{6}\right)$. In order to obtain $y^{+} \approx 1$, the required spacing at the wall is approximately $2 \times 10^{-6}$; however, $y^{+} \approx 10$ allows spacing an order of magnitude larger. Although not shown here, the $y^{+}$ obtained from actual computations on the baseline grid at an angle of attack of $22.36^{\circ}$ is approximately one over the first 20 percent of the airfoil and then drops to about $1 / 2$ afterwards. Values of $y^{+}$ for the second mesh are slightly above 10 for the first 20 percent and drop to around 5 for most of the remainder of the element. Because the sublayer for a turbulent boundary layer extends to a $y^{+}$of approximately 10 , essentially no points exist in this region over the first 20 percent of the airfoil for this mesh.

Figure 11 shows that inadequate spacing at the wall drastically affects the pressure distribution on the flap; hence, the other elements are effected as well. Inspection of the velocity profiles (not shown) reveals that inadequate spacing near the wall leads to an artificial thickening of the computed boundary layer on the main element. As discussed in reference [3], a thick boundary layer on the main element acts to suppress the loading on the flap. Therefore, the effect of the artificially thickened boundary layer is to artificially suppress the loading on the flap.

Numerical experiments indicate that inadequate wall spacing has little effect on the pressure distributions at lower angles of attack, such as $8.23^{\circ}$. It is primarily at higher angles where the wall spacing has been observed to be critical. Note that for single element airfoils, the effect of wall spacing is not as dramatic as it is for multielement configurations because the wake does not impinge on aft elements. Also, achieving a $y^{+}$of 1 is not a necessary requirement; typically, values similar to those used for algebraic turbulence models $\left(y^{+} \approx 3\right.$ ) should be sufficient [14], [15].

A summary of computed and experimental lift coefficients at Reynolds numbers of both 5 and 9 million are shown in figure 12. Here, the lift versus angle of attack is shown for the full configuration, as well as for the individual elements. The lift for each of the individual elements is obtained from pressure integration and is not corrected for wind tunnel wall effects. The total lift, on the other hand, is also computed from pressure integration, but has been corrected for wall interference.

As seen in the figure, the lift agreement is good for the uncorrected data on each element whereas the total lift (corrected) for the configuration is overpredicted in the computations. Although not shown, improved agreement between the computations and the experiment is obtained by using the lift from the force balance because it is slightly higher. For the computations, the angle of attack for maximum lift is not accurately predicted for either Reynolds number; however, the overall trend is well represented. The experiment and computations both obtain higher lift for a Reynolds number of 9 million over that of 5 million, and the main element begins to lose lift before either of the other elements.

A comparison of velocity profiles for Reynolds numbers of 5- and 9 million are shown in figure 13 for $\alpha=16.3^{\circ}$. Only small differences are seen over the main element, although the boundary layer at 9 million appears to be slightly thinner than at 5 million. Over the flap, more differences are apparent; the computations at the lower Reynolds number show lower velocities than at a Reynolds number of 9 million. Although the results from the grid convergence studies indicate that more refinement is required to adequately resolve the wake emanating from the slat which persists to the back of the flap, the overall trends in the velocity profiles with variations in Reynolds number are well captured.

The last case considered from this data set is a comparison of the computations and experiment between the $30 \mathrm{P}-30 \mathrm{~N}$ and the 30P-30AG configurations. Recall from the description of the wind tunnel tests that these configurations differ only in the flap rigging, as shown in figure 3 . Because the tests for the 30P-30AG 
configuration were conducted with only the two-segment flap, the data shown below for both the 30P-30N and the 30P-30AG are from this same test.

A comparison of the lift coefficients versus angle of attack for these configurations is shown in figure 14 for a Reynolds number of 9 million. In both the computations and the experiment, the $30 \mathrm{P}-30 \mathrm{~N}$ configuration attains higher lift coefficients than the $30 \mathrm{P}-$ 30AG. Note that the agreement between the computed lift and the experimental data for the $30 \mathrm{P}-30 \mathrm{~N}$ configuration is somewhat improved over that shown previously in figure 12 . This is because the data for the two-segment flap is used for the current figure. Careful inspection of figures 12 and 14 indicates that differences in the experimentally obtained lift between the one-and two-segment flap appear to start at about $20^{\circ}$. This difference may be due to slight differences in the model geometry or because the flow may no longer be two dimensional at this angle of attack.

A comparison of computed and experimental pressure distributions is shown in figure 15 for an angle of attack of $16.3^{\circ}$. The agreement between the computations and experiment is good for all elements. Although little difference due to the flap rigging is apparent on the slat and the main element, the flap shows a higher suction peak for the $30 \mathrm{P}-30 \mathrm{~N}$ configuration than for 30P-30AG.

\section{Conclusions and Recommendations}

A two-dimensional unstructured Navier-Stokes code has been utilized for computing the flow around multielement airfoil configurations. Comparisons are shown for a landing configuration with an advanced-technology flap for angles of attack up to the maximum-lift condition. A systematic grid convergence study has been conducted to assess the inaccuracies in the computations caused by inadequate grid resolution. Below maximum lift, pressure distributions are adequately resolved by using approximately 50,000 nodes. However, at high angles of attack, further grid refinement is required to obtain suitable levels of grid convergence for velocity profiles. This could be achieved by continuing to refine the mesh in a systematic manner, or possibly through the use of adaptive gridding or higher-order methods. The grid studies further indicate that care must be taken in obtaining accurate resolution of the wall boundary layers on upstream elements by using sufficiently small spacing of grid points. The use of a grid with inadequate wall spacing $\left(y^{+}=O(10)\right)$ results in an artificially thick boundary layer on the main element that severely effects the loading on the flap and hence the entire configuration.

Comparisons for the advanced flap configuration between computed and experimental pressure distributions are made for two flap riggings. In addition, lift coefficients and velocity profiles are compared for Reynolds numbers of 5- and 9 million. In general, the trends due to variations in rigging and Reynolds numbers are predicted well by the computations although the angle of attack for maximum lift is overpredicted.

\section{Acknowledgments}

The authors would like the thank the Aerodynamics Research and Technology group at the Douglas Aircraft Company for providing experimental data.

\section{References}

[1] Smith, A. M. O., "High-Lift Aerodynamics," AIAA Journal of Aircraft (37th Wright Brothers Lecture), vol. 12, June 1975.

[2] Woodward, D. S., and Lean, D. E., "Where is High-Lift Today?- A Review of Past UK Research Programmes," $\mathrm{Pa}$ per presented at AGARD meeting on high-lift aerodynamics. AGARD CP-415, Oct. 1992.

[3] Meredith, P. T., "Viscous Phenomena Affecting High-Lift Systems and Suggestions for Future CFD Development," Paper number 19 presented at AGARD meeting on high-lift aerodynamics. AGARD CP-415, Oct. 1992.

[4] Kirkpatrick, D., and Woodward, D., "Priorities for High-Lift Testing in the 1990's," AIAA 90-1413, June 1990.

[5] Lynch, F. T., "Experimental Necessities for Subsonic Transport Configuration Development," AIAA 92-0158, Jan. 1992.

[6] Warren, G. P., Anderson, W. K., Thomas, J. L., and Krist, S. L., "Grid Convergence for Adaptive Methods," AIAA 911592, June 1991.

[7] Mavriplis, D. J., "Turbulent Flow Calculation Using Unstructured and Adaptive Meshes," International Journal for Num. Meth. in Fluids, vol. 13, pp. 1131-1152, 1991.

[8] Barth, T. J., "Numerical Aspects of Computing Viscous High Reynolds Number Flows on Unstructured Meshes," AIAA 91-0721, 1991.

[9] Venkatakrishnan, V., and Mavriplis, D. J., "Implicit Solvers for Unstructured Meshes," AIAA 91-1537CP, 1991.

[10] Marcum, D. L., and Agarwal, R., "A Three-Dimensional Finite Element Navier-Stokes Solver with k- $\epsilon$ Turbulence Model for Unstructured Grids," AIAA 90-1652, 1990.

[11] Anderson, W. K., and Bonhaus, D. L., "An Implicit Upwind Algorithm for Computing Turbulent Flows on Unstructured Grids," Computers and Fluids, vol. 23, no. 1, pp. 1-21, 1994.

[12] Anderson, W. K., and Bonhaus, D. L., "Navier-Stokes Computations and Experimental Comparisons for Multielement Airfoil Configurations," AIAA 93-0645, 1993.

[13] Roe, P., "Approximate Riemann Solvers, Parameter Vectors, and Difference Schemes," J. of Comp. Phys., vol. 43, pp. 357-372, 1981.

[14] Baldwin, B. S., and Barth, T. J., "A One-Equation Turbulence Transport Model for High Reynolds Number Wall Bounded Flows," NASA Technical Memorandum 102847, Aug. 1991.

[15] Spalart, P. R., and Allmaras, S. R., "A One-Equation Turbulence Model for Aerodynamic Flows," AIAA 92-0439, 1991.

[16] Stainback, P. C., McGhee, R. J., Beasley, W. D., and Morgan, H. L., "The Langley Research Center's Low Turbulence Pressure Tunnel," AIAA 86-0762, 1986.

[17] Paschal, K., Goodman, W., McGhee, R., and Walker, B., "Evaluation of Tunnel Sidewall Boundary-Layer-Control Systems for High-Lift Testing," AIAA 91-3243, 1991.

[18] Lin, J. C., Robinson, S., McGhee, R. J., and Valarezo, W. O., "Separation Control on High Reynolds Number Multielement Airfoils," AIAA 92-2636, 1992.

[19] Valarezo, W. O., Dominik, C. J., McGhee, R. J., and Goodman, W. L., "High Reynolds Number Configuration Development of a High-Lift Airfoil," Paper 10-1, AGARD meeting in high-lift aerodynamics, Oct. 1992. 


\section{ANDERSON ET AL.: NAVIER-STOKES COMPUTATIONS}

[20] Chin, V., Peters, D. W., Spaid, F. W., and McGhee, R. J., "Flowfield Measurements About a Multi-Element Airfoil at High Reynolds Numbers," AIAA 93-3137, 1993.

[21] Mavriplis, D., "Adaptive Mesh Generation for Viscous Flows using Delaunay Triangulation,” J. of Comp. Phys., vol. 90, pp. 271-291, 1990.

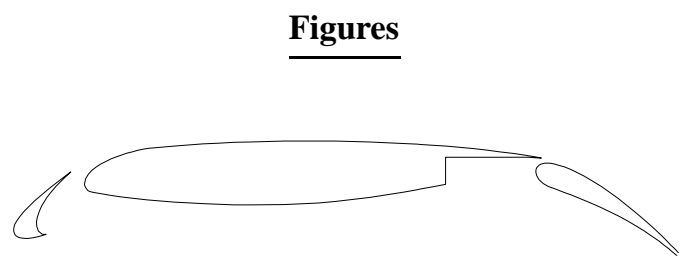

Figure 1. Geometry for three-element airfoil 30P-30AG.

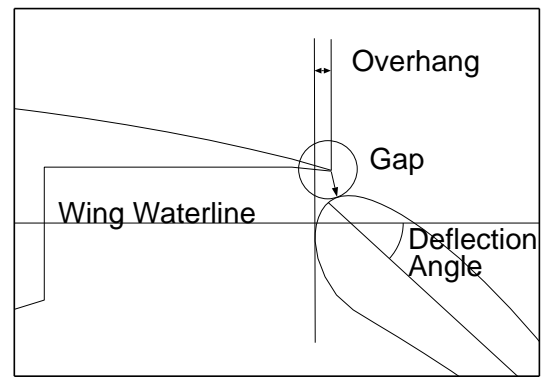

Figure 2. Definition of gap and overhang for flap.

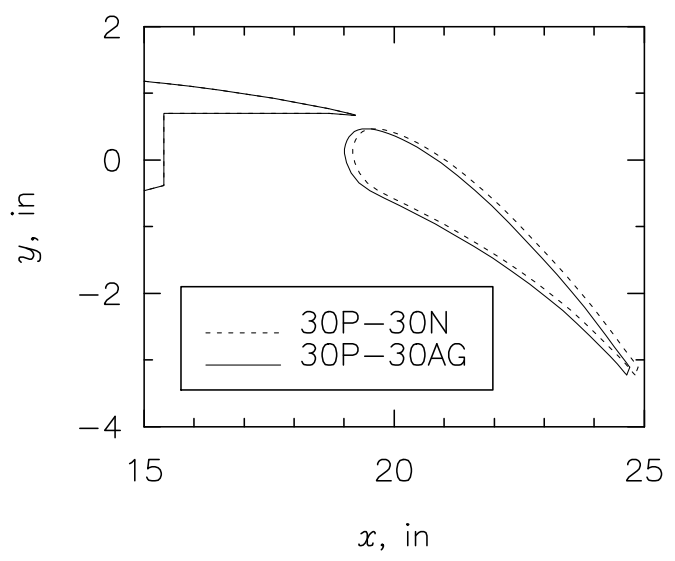

Figure 3. Differences in flap rigging for the $30 \mathrm{P}-30 \mathrm{~N}$ and $30 \mathrm{P}-30 \mathrm{AG}$ configurations.
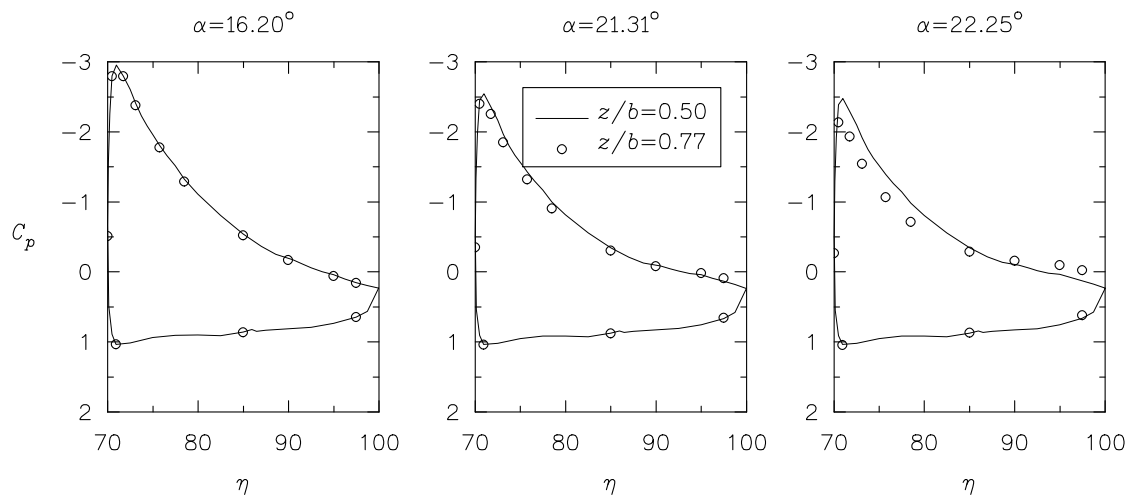

Figure 4. Experimental pressure distributions at two spanwise locations for several angles of attack.

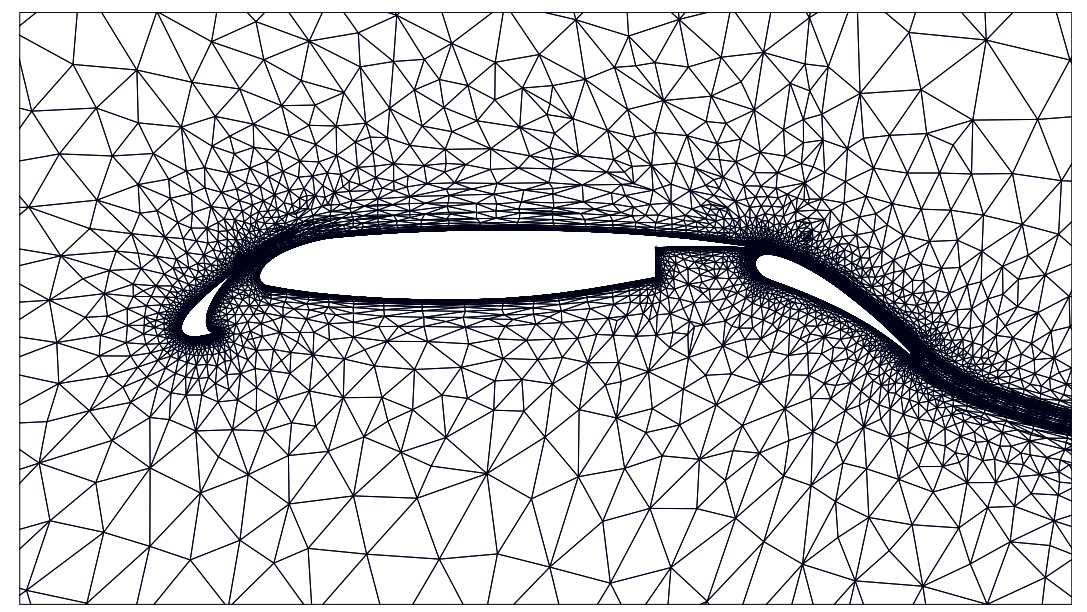

Figure 5. View of sample unstructured grid for three-element airfoil. 
ANDERSON ET AL.: NAVIER-STOKES COMPUTATIONS
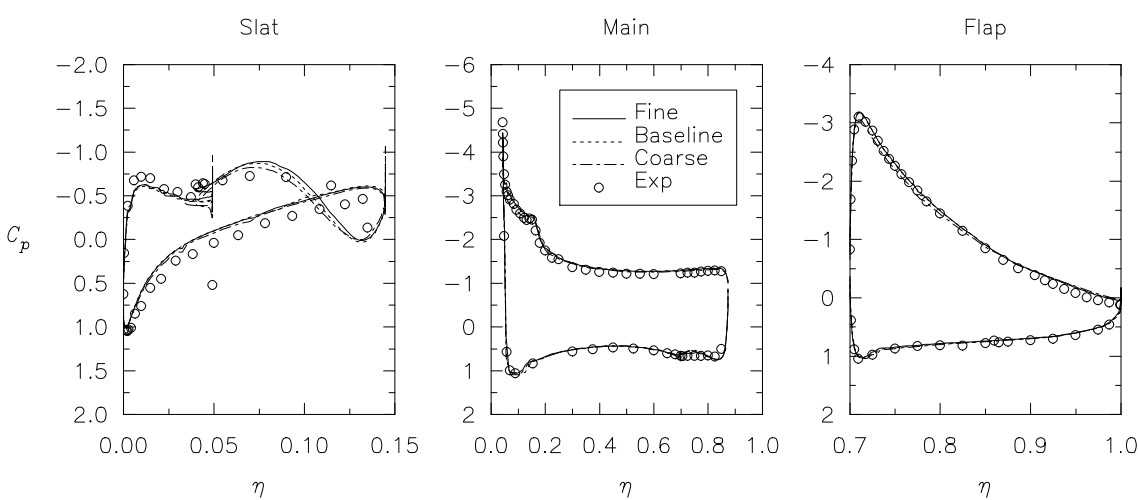

Figure 6. Computed pressure distributions on the coarse, baseline, and fine grids for $30 \mathrm{P}-30 \mathrm{~N}$ with $M_{\infty}=0.2, R_{e}=9 \times 10^{6}, \alpha=-0.03^{\circ}$.
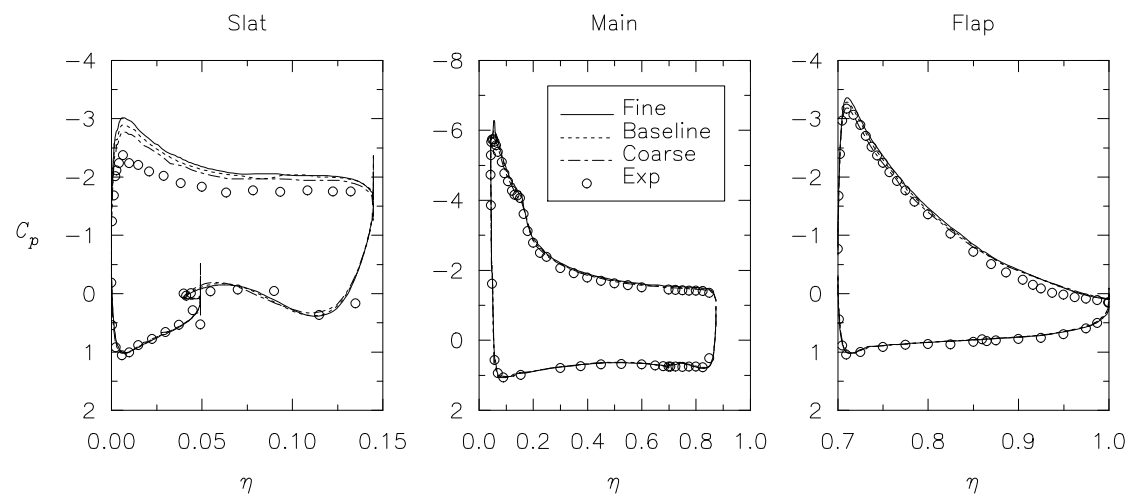

Figure 7. Computed pressure distributions on the coarse, baseline, and fine grids for $30 \mathrm{P}-30 \mathrm{~N}$ with $M_{\infty}=0.2, R_{e}=9 \times 10^{6}, \alpha=8.23^{\circ}$.
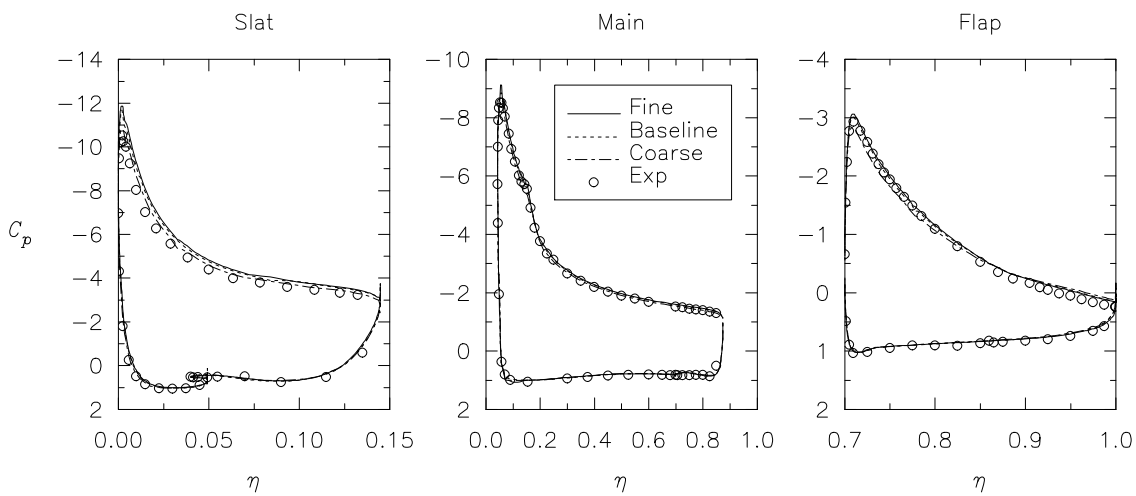

Figure 8. Computed pressure distributions on the coarse, baseline, and fine grids for $30 \mathrm{P}-30 \mathrm{~N}$ with $M_{\infty}=0.2, R_{e}=9 \times 10^{6}, \alpha=16.30^{\circ}$.
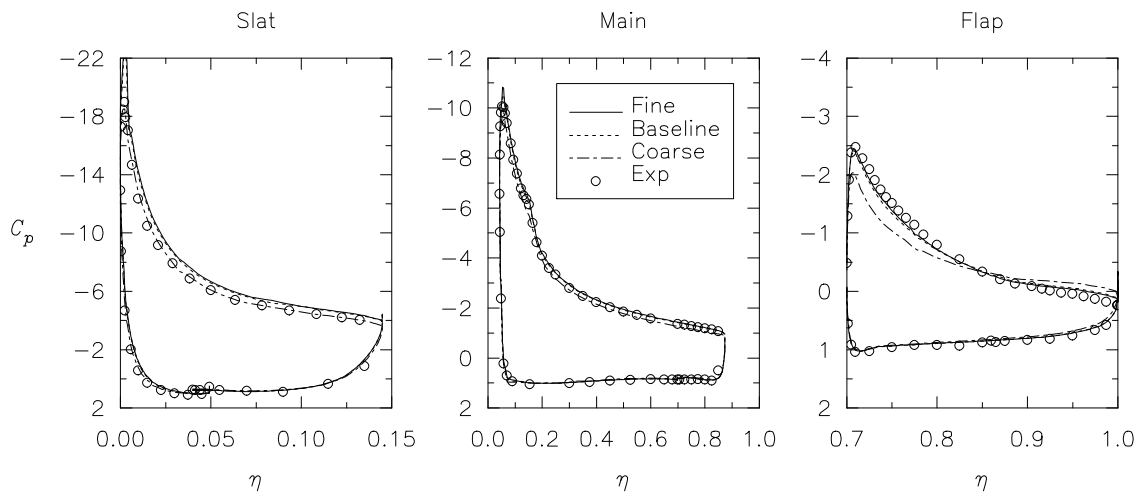

Figure 9. Computed pressure distributions on the coarse, baseline, and fine grids for 30P-30N with $M_{\infty}=0.2, R_{e}=9 \times 10^{6}, \alpha=22.36^{\circ}$. 
ANDERSON ET AL.: NAVIER-STOKES COMPUTATIONS
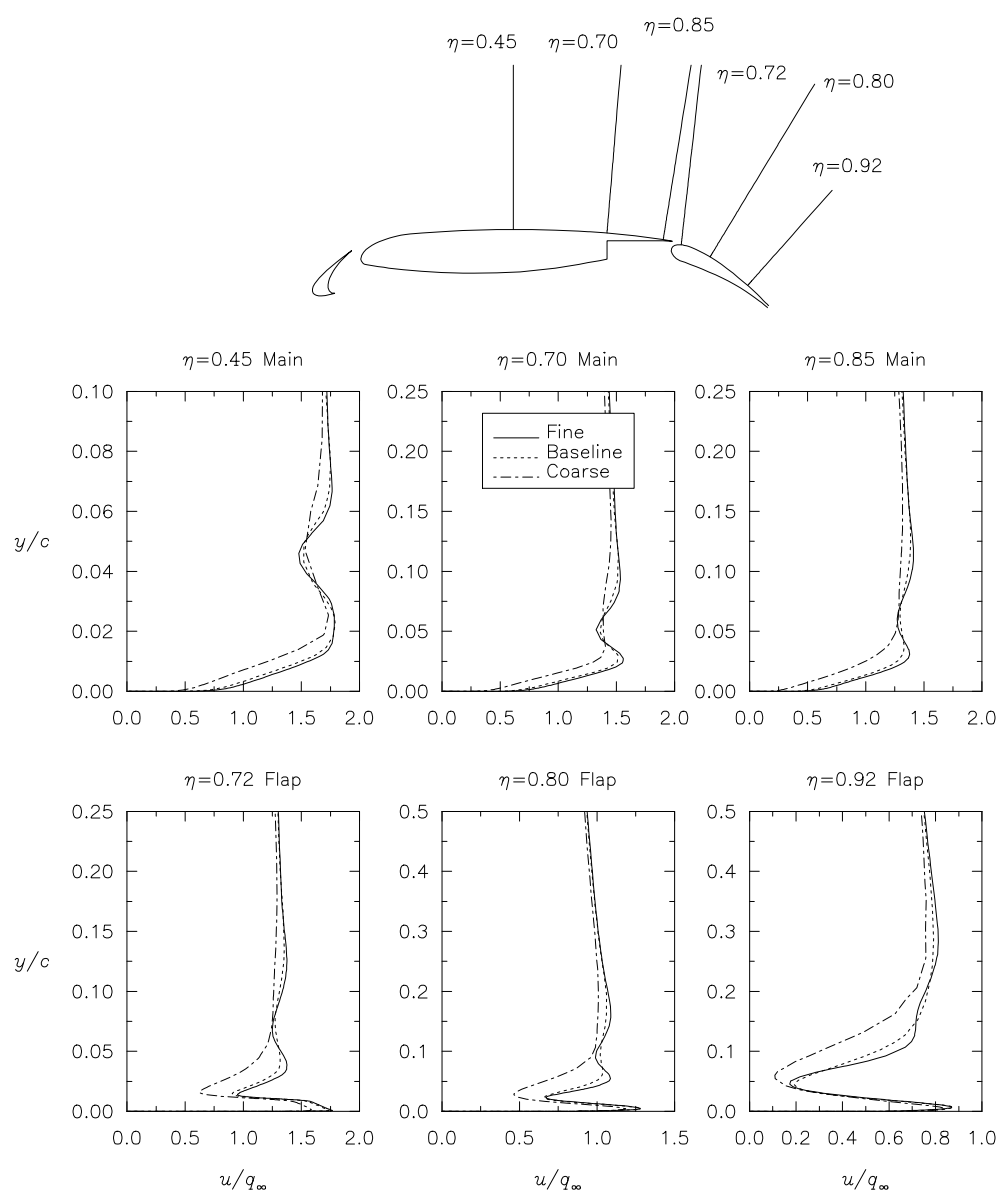

Figure 10. Computed velocity profiles on the coarse, baseline, and fine grids for 30P-30N with $M_{\infty}=0.2, R_{e}=9 \times 10^{6}, \alpha=22.36^{\circ}$.
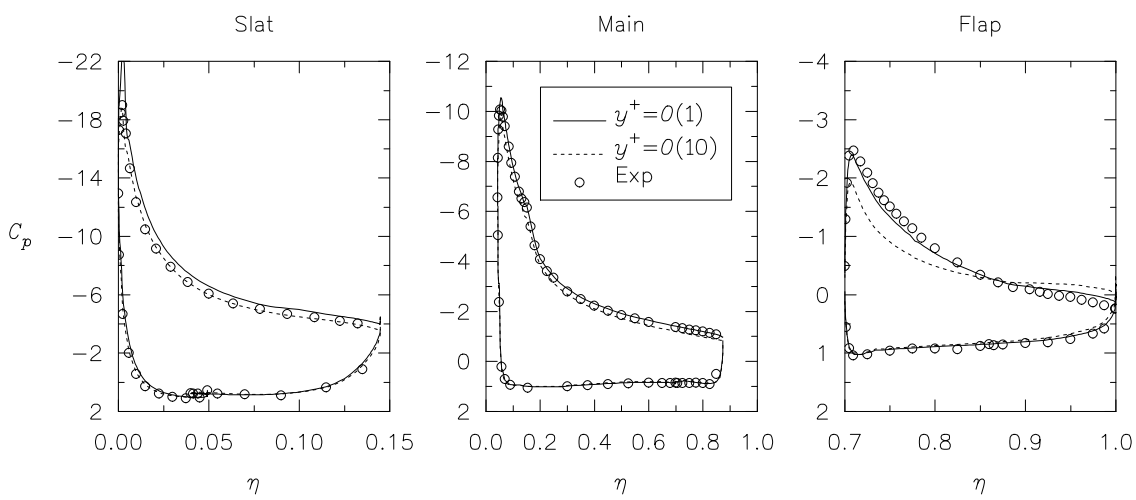

Figure 11. Comparison of pressure distributions on grids with $y^{+} \approx 1$ and $y^{+} \approx 10$ for $30 \mathrm{P}-30 \mathrm{~N}$ with $M_{\infty}=0.2, R_{e}=9 \times 10^{6}, \alpha=22.36^{\circ}$. 
ANDERSON ET AL.: NAVIER-STOKES COMPUTATIONS

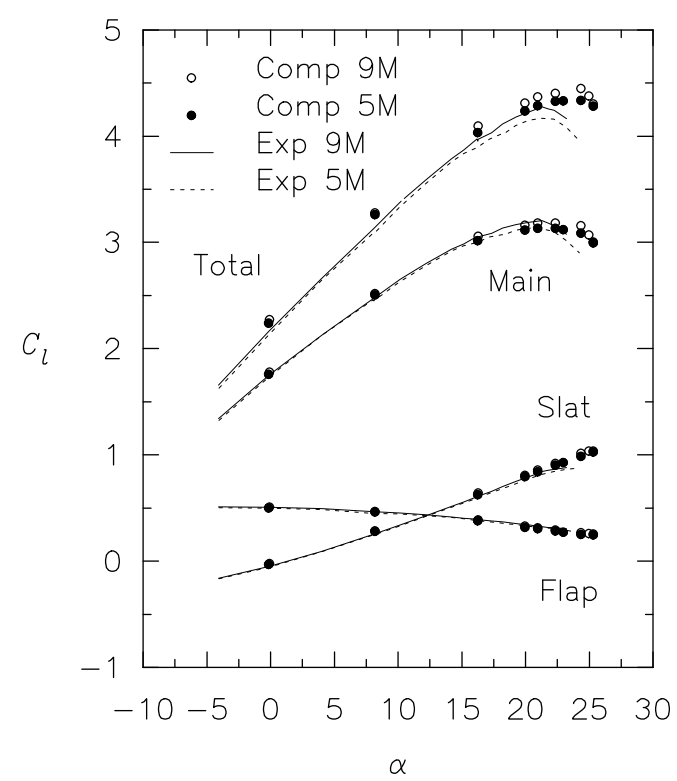

Figure 12. Experimental and computational lift versus angle of attack for $R_{e}=5 \times 10^{6}$ and $R_{e}=9 \times 10^{6}$ for $30 \mathrm{P}-30 \mathrm{~N}$ with $M_{\infty}=0.2$.
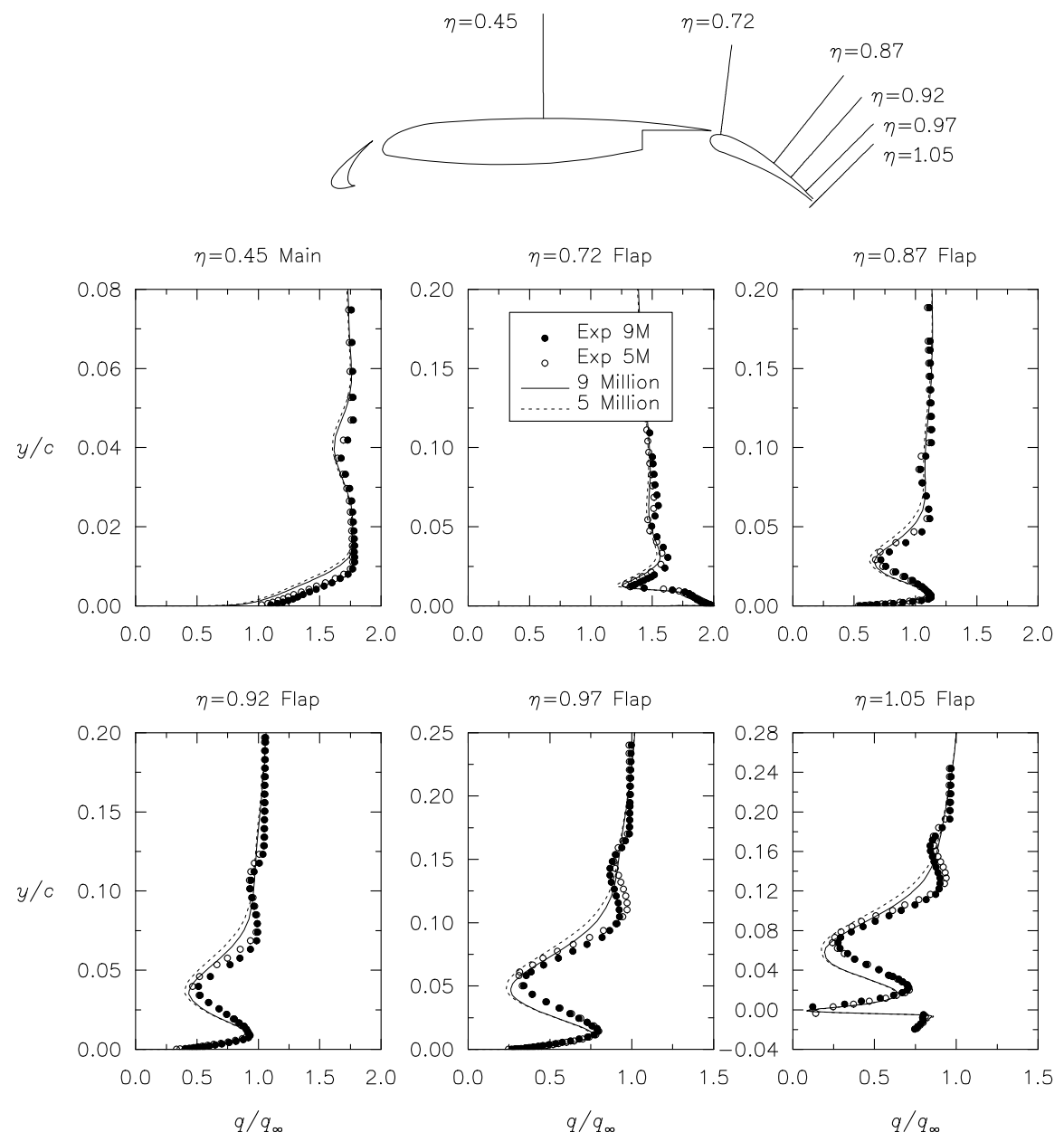

Figure 13. Comparison of velocity profiles at Reynolds numbers of $R_{e}=5 \times 10^{6}$ and $R_{e}=9 \times 10^{6}$ for $30 \mathrm{P}-30 \mathrm{~N}$ with $M_{\infty}=0.2, \alpha=16.3^{\circ}$. 
ANDERSON ET AL.: NAVIER-STOKES COMPUTATIONS

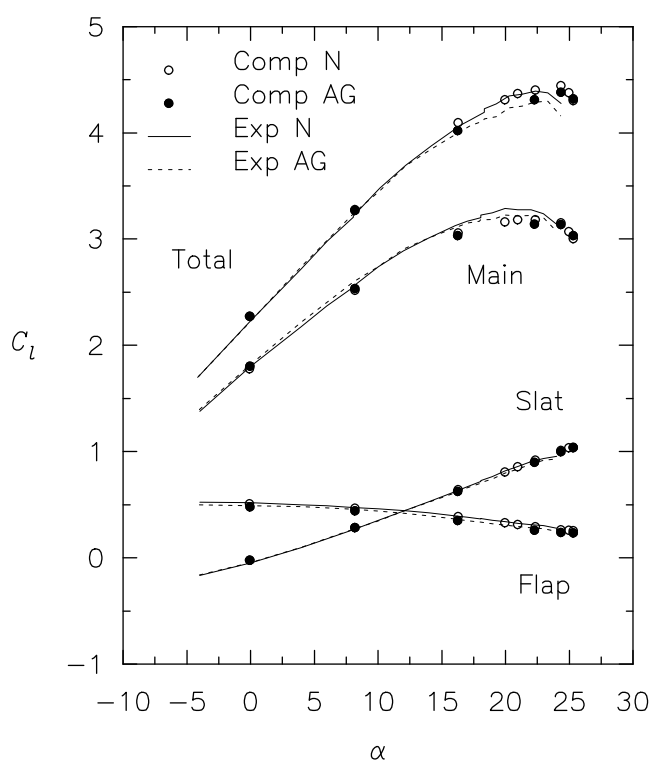

Figure 14. Comparison of computational and experimental lift for the $30 \mathrm{P}-30 \mathrm{~N}$ and 30P-30AG configurations with $M_{\infty}=0.2, R_{e}=9 \times 10^{6}$.
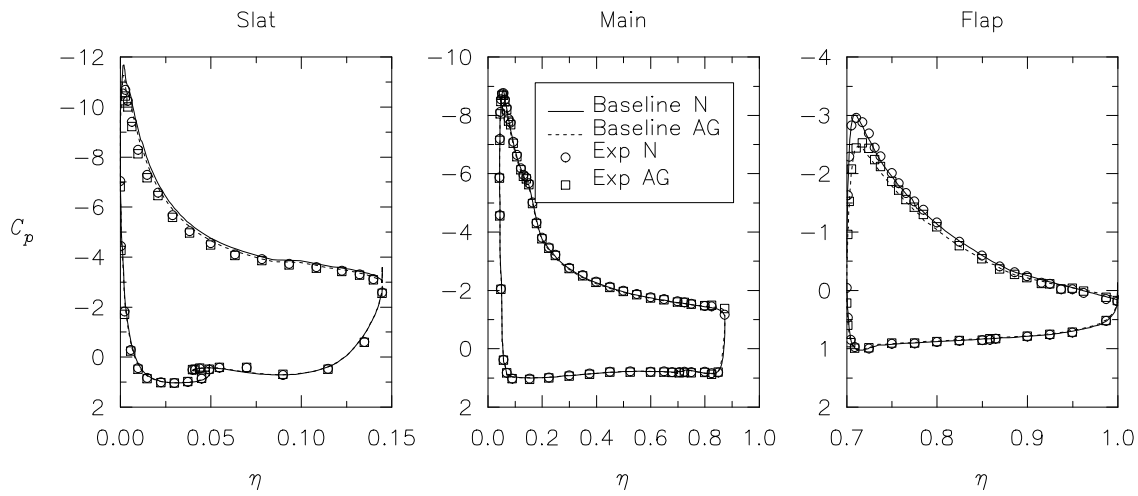

Figure 15. Comparison of computational and experimental pressure distributions for the 30P-30N and 30P-30AG configurations with $M_{\infty}=0.2, R_{e}=9 \times 10^{6}, \alpha=16.3^{\circ}$. 